\title{
Evaluation of Antimicrobial Susceptibility Among Acintobacter baumannii by E-Test Method at Khatam-Al-Anbia Hospital During 2013 -
}

\section{5}

\author{
Hadi Kazemi, ${ }^{1}$ Davood Yadegarynia, ${ }^{2}$ Sara Rahmati Roodsari, ${ }^{2,}{ }^{*}$ and Zahra Arab-Mazar ${ }^{2}$ \\ ${ }^{1}$ Department of Pediatrics, School of Medicine, Shahed University, Tehran, Iran \\ ${ }^{2}$ Infectious Diseases and Tropical Medicine Research Center, Shahid Beheshti University of Medical Sciences, Tehran, Iran \\ "Corresponding author: Sara Rahmati Roodsari, Infectious Diseases and Tropical Medicine Research Center, Shahid Beheshti Universtiy of Medical Sciences, Tehran, Iran. \\ E-mail: s_r_r85@yahoo.com
}

Received 2016 April 24; Revised 2016 June 13; Accepted 2017 January 09.

\begin{abstract}
Background: Nosocomial infections are one of the health problems of modern societies, which are rising with unusual organisms. Acintobacter, which is the main cause of nosocomial infections such as pneumonia and nosocomial pneumonia, is caused by mechanical ventilation. Acinetobacter species are becoming resistant to antibiotics. One the most important agent of nosocomial infections with high mortality is infections by Acinetobacter baumannii which is Gram- negative opportunistic Coccobacilli. Treatment in these infections is difficult and sometimes impossible, due to multidrug resistance in strains isolated from nosocomial infections.

Objectives: The aim of the current study was to evaluate antibiotic resistance in A. baumannii isolates Khatam-Al-Anbia Hospital, Tehran, Iran.

Methods: In this cross-sectional study 100 of Acintobacter baumannii were isolated from hospitalized patients during 2013-2015 in Khatam-Al-Anbia hospital in Tehran. In this study samples of A. baumannii isolated from trachea, blood, urine, sputum and wound samples of patients bedridden in Intensive care unit (ICU) wards. Antimicrobial susceptibility and minimum inhibitory concentration (MIC) were determined by E-test methods. We used descriptive statistics to analyze the data by using SPSS 21 software.

Results: A total of 100 A. baumannii were isolated from clinical samples. The organism was resistant to rifampicin (46\%), gentamicin (67\%), meropenem (100\%), piperacilin (98\%), colistin (0\%), and ceftazidin (96\%).

Conclusions: The antibiotic resistance against most of the antibiotics especially meropenem is very high in this study. Moreover, colistin was most effective antibiotic to be used in A. baumannii infections. Colistin is the best choices for treatment of Acinetobacter.
\end{abstract}

Keywords: Acintobacter baumannii, Resistance, Susceptibility, E-Test Method

\section{Background}

Acinetobacter baumannii is a ubiquitous, nonfermenting, aerobic Gram-negative bacterium with intrinsic resistance to multiple antimicrobial agents $[1,2]$. Nowadays the nosocomial infections due to Acintobacter baumannii have notably increased, although in past decades, these pathogen infections have been sporadically identified in hospitals. Due to its multidrugresistance, morbidity and mortality in healthcare setting, this pathogen becomes important [3]. Acinetobacter baumannii can be detected in different environmental sources such as soil and foods. This pathogen sometimes can colonize the kin of healthy human, typically at a lowdensity and for short-term duration. Colonization of other body parts like the throat, nares, and the intestinal tract , was seen rarely in healthy human as well $[4,5]$. Acinetobacter infections, mostly progress towards bacteremia, septicemia also lower respiratory tract involvement with
Acinetobacter, are the most common source of infection in regards to Acinetobacter progression towards bacteremia and septicemia [6].

Acintobacer baumannii pathogen has known as important and common agent which is causing nosocomial pneumonia and bacteremia worldwide among patient who admitted in the intensive care unit(ICU) [7-9], which is isolated from skin, soft tissue, and urinary tract infection, and secondary meningitis over the past decades.

Over few last decades, the incidence of A. baumannii infections has increased, that may associated to increasing in the proportion of the susceptible population because of advancements in medical support of patients who are in critical situation $[10,11]$.

The rate of mortality of A. baumannii is around $7.8 \%$ to $23 \%$ for hospitalized patients with infection and the rate was $10 \%$ to $43 \%$ among ICU admitted patients [12]. If the infection caused by isolates with multiple classes of antimicrobial agents resistance, it seems that outcomes of this pa- 
tients be poorer [13].

The aim of this research was to evaluate the antibiotic resistance in Acintobacer baumannii by using E-test at the Khatam-Al-Anbia hospital during 2013 - 2015.To deal with the above-mentioned issues, our objective was to inform about the antibiotic resistance in every region, to choose the correct treatment, and to take the necessary steps in preventing further resistance thereby reducing morbidity and mortality.

\section{Methods}

This is a cross-sectional study. During the project, the samples containing isolated Acintobacer baumannii were sent from different parts of the Khatam-Al-Anbia hospital in Tehran capital of Iran during 2013 - 2015. A. baumannii were examind on isolates of patients admitted at least 48 hours in the hospital. The specimens of patients included respiratory tube, urine, wound, and blood. The identification of A. baumannii and resistant pattern was done by using conventional bacteriological method and clinical laboratory and standards Institute (CLSI), respectively.

For all specimens, laboratory tests were performed including MacConkey and blood agar plates as routin, trypticase soy broth (TSB), and sub-cultured on chocolate agar for blood specimens, and chocolate agar for specimens other than urine. All of the suspected colonies were assessed by gram-staining, colonial morphology, negative oxidase, and other biochemical reactions [14].

E-test method which is recommended by clinical laboratory and standard institute (CLSI) was used to evaluate antimicrobial susceptibility of the isolate organisms. MICs (minimum inhibitory concentrations) were determined by the E-test method according to the manufacturer's guidelines (AB Biodisk). A suspension of each isolate in Mueller-Hinton broth, adjusted to the density of a 0.5 McFarland standard, was swabbed in three directions to ensure uniform growth onto Mueller-Hinton agar plates. Once the agar surface was completely dry, E-test strips were applied to each plate with sterile forceps, and the plates were incubated at $35^{\circ} \mathrm{C}$ for 16 to 20 hours. The MIC was read where inhibition of growth intersected the E-test strip. When small colonies grew within the zone of inhibition or a haze of growth occurred around MIC end points, the highest MIC intersect was recorded.

The ethics committee of School of Medicine, Shahid Beheshti University of Medical Sciences was approved this study. Statistical analysis was performed by using SPSS 21 software (SPSS Inc., Chicago, IL). A two-tailed P-value $<0.05$ was considered statistically significant.

\section{Results}

Overall, 100 strains of A. baumannii were isolated from the hospitalized patients between 2013 to 2015 from ICU ward of Khatam-ol-Anbia Hospital, Tehran, Iran. The mean age of patients was 71.97 years in which $68(68 \%)$ patients were men and 32 (32\%) were female. Distribution of clinical samples were respiratory tract was the most common place $39 \%$ of Acinetobacter isolation including tracheal secretion (32.3\%); sputum (6.7\%); other common sites were blood (9.4\%); wound (1.9\%); CSF (13.2\%); and catheter(3.8\%) urine (32.7\%).

The rates of resistance were $46 \%$ for rifampicin, $67 \%$ for gentamicin, $100 \%$ for meropenem, $98 \%$ for piperacilin, $0 \%$ for colistin, and $96 \%$ for ceftazidin.

\section{Discussion}

In this study, the antibiotic resistance against most of the antibiotics especially meropenem is very high. Moreover colistin was most effective antibiotic to be used in A. baumannii infections. Colistin is the best choices for treatment of Acinetobacter.

Acinetobacter baumannii is a pathogen that is opportunistic and involved in a large number of hospitalacquired infections and Couse of increasing mortality and morbidity [15]. Nowadays, nosocomial infection with multidrug-resistant Acinetobacter is important problems in the world, which are facing wide spectrum antibiotics, have become resistant. Since the 1970s, the spread of multidrug-resistant (MDR) Acinetobacter strains have become an increasing cause of concern among critically ill, hospitalized patients, and subsequent epidemics [4].

Today we are facing with the problem of dominance of Acinetobacter baumannii [16] which our study also reached to the same result. Same as other studies our research showed that Acinetobacter has the most relation with respiratory system and most of the positive samples for Acinetobacters were obtained from respiratory secretions and the respiratory tract is the main source of Acinetobacter infection $[17,18]$.

In the present study drug resistance in A. baumannii isolates from ICU wards of hospital was very high, especially against meropenem piperacilin, and ceftazidin rate of $100 \%, 98 \%$, and $96 \%$, respectively. In Iran, many researches have been done about drug resistance of $\mathrm{A}$. baumannii during recent years and they have found similar high resistant rate to most of the antibiotics [19, 20] likely to our study,in 2008, Feizabadi et al. investigated that prevalence of susceptibility of A. baumannii to imipenem, meropenem, piperacillin-tazobactam and 
amikacin percentage of $50.7 \%, 50 \%, 42.1 \%$ and $38.2 \%$, respectively. In 2009 Morovat et al. reported susceptibility of A. baumannii to cefotaxime, imipenem, meropenem, piperacillin, piperacillin-tazobactam, and tigecycline rates of $7.5 \%, 42.5 \%, 42.5 \%, 21.2 \%, 28.7 \%$ and $91.2 \%$ using E-test method [21].

Inappropriate prescription of highly used antibiotics for treatment of infections, has caused the emergence of multi-drug resistant pathogens including Acinetobacter, hence research results show the increasing resistance of Acinetobacter to highly used antibiotics [22, 23].

The studies which have been done in Iran and other countries have reporeted a pattern of increased antibiotic resistance, and we are facing to the emergence of multi-drug resistant (MDR) strains. This may because of prolonged hospital stays, irrational prescription of antibiotics, weakened immune systems because of underlying factors, and immune-system depressant drugs [24].

\subsection{Conclusion}

In conclusion, our study showed the antibiotic resistance of A. baumannii isolates in Khatam-Al-Anbia hospital during 2013 - 2015. It seems that the only way to manage this changing pattern is to approach it with rational broadspectrum drug prescriptions and treatment of A. baumannii infections. This can be performed by giving appropriate instructions in different way like having broad-spectrum drugs prescribed only by specialists in infectious diseases, in addition have mandatory consultations to prescribe antibiotics and control the rate of resistance.

\section{Acknowledgments}

The authors would like to acknowledge their gratitude to Dr. Azad who participated in data collection in KhatamAl-Anbia Hospital Laboratory. This study was financially supported by the grant No.6898 provided from Infectious Diseases and Tropical Medicine research center, Shahid Beheshti University of Medical Sciences.

\section{Footnotes}

Authors' Contribution: The core idea of this work came from Davood Yadegarynia and Dr. Hadi Kazemi. Dr. Sara Rahmati Roodsari and Zahra Arab-Mazar collected the data and acted as technical and material support.

Financial Disclosure: There are not any conflicts of interest.

Funding/Support: This study was financially supported by the grant No.6898 provided from Infectious Diseases and Tropical Medicine research center, Shahid Beheshti University of Medical Sciences.

\section{References}

1. Hancock RE. Resistance mechanisms in Pseudomonas aeruginosa and other nonfermentative gram-negative bacteria. Clin Infect Dis. 1998;27 Suppl 1:93-9. [PubMed: 9710677].

2. Liassine N. [Problems of antibiotic-resistance Gram negative pathogens in the hospital environment]. Schweiz Med Wochenschr. 2000;130(50):1930-6. [PubMed: 11189438].

3. Fresnadillo-Martinez MJ, Garcia-Merino E, Garcia-Sanchez E, Martindel Rey A, Rodriguez-Encinas A, Rodriguez-Sanchez G, et al. [Prevention of an outbreak of Acinetobacter baumannii in intensive care units: study of the efficacy of different mathematical methods]. Rev Esp Quimioter. 2015;28(1):10-20. [PubMed: 25690140].

4. Dijkshoorn L, Nemec A, Seifert H. An increasing threat in hospitals: multidrug-resistant Acinetobacter baumannii. Nat Rev Microbiol. 2007;5(12):939-51. doi: 10.1038/nrmicro1789. [PubMed: 18007677].

5. Chu YW, Leung CM, Houang ET, Ng KC, Leung CB, Leung HY, et al. Skin carriage of acinetobacters in Hong Kong. J Clin Microbiol. 1999;37(9):2962-7. [PubMed: 10449482].

6. Yadegarinia D, Abedy SH, Gachkar L, Rahmati Roodsari S. Prevalence and drug resistance of Acinetobacter baoumanii in ICU of teaching hospital.J Appl Environ Biol Sci. 2013;3(9):22-7.

7. Gaynes R, Edwards IR, National Nosocomial Infections Surveillance S. Overview of nosocomial infections caused by gram-negative bacilli. Clin Infect Dis. 2005;41(6):848-54. doi: 10.1086/432803. [PubMed: 16107985].

8. Kanafani ZA, Kara L, Hayek S, Kanj SS. Ventilator-associated pneumonia at a tertiary-care center in a developing country: Incidence, microbiology, and susceptibility patterns of isolated microorganisms • Infect Control Hosp Epidemiol. 2003;24(11):864-9. doi: 10.1086/502151.

9. Yadegarynia D, Azad MK, Gachkar L, Roodsari SR, Arab-Mazar Z. Drug resistance of acinetobacter in selected hospitals. Novel biomed. 2015;3(3):103-10.

10. Bergogne-Berezin E, Towner KJ. Acinetobacter spp. as nosocomial pathogens: microbiological, clinical, and epidemiological features. Clin Microbiol Rev. 1996;9(2):148-65. [PubMed: 8964033].

11. Wisplinghoff H, Bischoff T, Tallent SM, Seifert H, Wenzel RP, Edmond MB. Nosocomial bloodstream infections in US hospitals: analysis of 24,179 cases from a prospective nationwide surveillance study. Clin Infect Dis. 2004;39(3):309-17. doi: 10.1086/421946. [PubMed: 15306996].

12. Falagas ME, Rafailidis PI. Attributable mortality of Acinetobacter baumannii: no longer a controversial issue. Crit Care. 2007;11(3):134. doi: 10.1186/cc5911. [PubMed: 17543135].

13. Yadegarynia D, editor. Isolation of 100 multi drug resistant (mdr) acinetobacter baumannii in two tertiary care hospitals in tehran, iran. 46th Annual Meeting. 2008; Idsa.

14. Forbes BA, Sahm DF, Weissfeld AS. Study guide for Bailey and Scott's diagnostic microbiology. Elsevier Health Sciences; 2016.

15. Kempf M, Rolain JM. Emergence of resistance to carbapenems in Acinetobacter baumannii in Europe: clinical impact and therapeutic options. Int J Antimicrob Agents. 2012;39(2):105-14. doi: 10.1016/j.ijantimicag.2011.10.004. [PubMed: 22113193].

16. Henwood CJ, Gatward T, Warner M, James D, Stockdale MW, Spence RP, et al. Antibiotic resistance among clinical isolates of Acinetobacter in the UK, and in vitro evaluation of tigecycline (GAR-936). J Antimicrob Chemother. 2002;49(3):479-87. [PubMed: 11864948].

17. Reddy T, Chopra T, Marchaim D, Pogue JM, Alangaden G, Salimnia H, et al. Trends in antimicrobial resistance of Acinetobacter baumannii isolates from a metropolitan Detroit health system. Antimicrob Agents Chemother. 2010;54(5):2235-8. doi: 10.1128/AAC.01665-09. [PubMed: 20211887] 
18. Wareham D, Bean D. Molecular typing of multi-drug resistant Acinetobacter baumannii from London hospitals. Int JInfect Dis. 2010;14:36. doi: 10.1016/j.ijid.2010.02.1566.

19. Feizabadi MM, Fathollahzadeh B, Taherikalani M, Rasoolinejad M, Sadeghifard N, Aligholi M, et al. Antimicrobial susceptibility patterns and distribution of blaOXA genes among Acinetobacter spp. Isolated from patients at Tehran hospitals. Jpn J Infect Dis. 2008;61(4):274-8. [PubMed: 18653968].

20. Peymani A, Nahaei MR, Farajnia S, Hasani A, Mirsalehian A, Sohrabi N, et al. High prevalence of metallo-beta-lactamase-producing acinetobacter baumannii in a teaching hospital in Tabriz, Iran.Jpn J Infect Dis. 2011;64(1):69-71. [PubMed: 21266761].

21. Taherikalani M, Fatolahzadeh B, Emaneini M, Soroush S, Feiz- abadi MM. Distribution of different carbapenem resistant clones of Acinetobacter baumannii in Tehran hospitals. New Microbiol. 2009;32(3):265-71. [PubMed: 19845108].

22. Lin MF, Lan CY. Antimicrobial resistance in Acinetobacter baumannii: From bench to bedside. World J Clin Cases. 2014;2(12):787-814. doi: 10.12998/wjcc.v2.i12.787. [PubMed: 25516853].

23. Abbasi F, Khalili-azad M, Asadi S, Yadegarynia D, Gholamin S, Gachkar L. Acinetobacter baumanii and nonbaumanii in iran, evaluation of antibiotic resistance. Iran J Public Health. 2014;43(2):35.

24. Yadegarynia D, Arab-Mazar Z. Evaluation of antimicrobial susceptibility among enterococcus species by e-test method at khatam-olanbia hospital during 2013 - 2014. Arch Clin Infect Dis. 2016;11(1) doi: 10.5812/archcid.29526. 